\title{
Clinical study of different doses of atorvastatin combined with febuxostat in patients with gout and carotid atherosclerosis
}

\author{
Zheng Zhang ${ }^{1}$, Ming-hua $X u^{2}$, \\ Feng-ju Wei ${ }^{3}$, Li-na Shang ${ }^{4}$
}

\begin{abstract}
Objective: To evaluate the efficacy of atorvastatin combined with febuxostat in the treatment of gout patients with carotid atherosclerosis and to observe the effects on serum tumor necrosis factor- $a$ (TNF- $\alpha$ ), interleukin-1B (IL-1B), and C-reactive protein (CRP) levels, carotid plaques, and the adverse reactions.

Methods: Seventy patients with gout and carotid atherosclerosis admitted to Affiliated Hospital of Hebei University from January 2014 to June 2017 were randomly divided into a treatment group and a control group. The treatment group received oral febuxostat $40 \mathrm{mg} /$ day combined with atorvastatin $40 \mathrm{mg} /$ day. The control group was given $40 \mathrm{mg} /$ day febuxostat combined with $20 \mathrm{mg} /$ day atorvastatin for 90 days. The effects of treatment on TNF- $a$, IL-1B, and CRP levels and carotid plaques of the patients were observed.

Results: After 90 days of treatment, serum TNF-a, IL-1B, and CRP levels, as well as HUA and total cholesterol (TC), decreased in both groups after treatment. There were significant differences observed $(p<0.05)$. The carotid artery plaques in the two groups were significantly smaller after treatment $(P<0.05)$. There was no significant difference in adverse reactions between the two groups ( $P>0.05)$.

Conclusion: Double doses of atorvastatin combined with febuxostat can effectively reduce uric acid to improve the inflammatory state in patients and reduce carotid plaques without increasing the incidence of adverse reactions.
\end{abstract}

KEYWORDS: Gout; Carotid atherosclerosis; Atorvastatin; Febuxostat.

doi: https://doi.org/10.12669/pjms.36.6.2945

How to cite this:

Zhang Z, Xu MH, Wei FJ, Shang LN. Clinical study of different doses of atorvastatin combined with febuxostat in patients with gout and carotid atherosclerosis. Pak J Med Sci. 2020;36(6):1334-1338. doi: https://doi.org/10.12669/pjms.36.6.2945

This is an Open Access article distributed under the terms of the Creative Commons Attribution License (http://creativecommons.org/licenses/by/3.0), which permits unrestricted use, distribution, and reproduction in any medium, provided the original work is properly cited.

1. Zheng Zhang,

Department of Rheumatology and Immunology,

2. Ming-hua Xu,

Department of Rheumatology and Immunology,

3. Feng-ju Wei,

Department of Traditional Chinese Medicine Department,

4. Li-na Shang,

Department of Ultrasound,

1-4: Affiliated Hospital of Hebei University,

Baoding, Hebei 071000, P. R. China.

Correspondence:

Li-na Shang,

Department of Ultrasound, Affiliated Hospital of Hebei University,

Baoding, Hebei 071000, P. R. China.

E-mail: bke434@163.com

* Received for Publication:

* Revision Received:

* Revision Accepted: *

May 22, 2020

July 23, 2020

July 28, 2020

\section{INTRODUCTION}

Gout is a group of diseases caused by a purine metabolism disorder that is closely related to hyperuricemia (HUA). Studies have shown that the prevalence of HUA in China is $12.08 \%,{ }^{1}$ which is significantly higher than it was 30 years ago, and that HUA is an independent risk factor for the occurrence and development of cardiovascular and cerebrovascular diseases, endocrine diseases and chronic kidney diseases. ${ }^{2}$ It has been reported that patients with gout continue to have microinflammation in their bodies even during the intermission period between attacks, and chronic low-grade inflammation is the basis of metabolic 
syndromes, such as obesity, hyperlipidemia, and atherosclerosis. ${ }^{3}$ A large number of studies have shown that rheumatoid arthritis, which is also a rheumatic immune disease, has a significantly increased risk of carotid plaque formation and cardiovascular events. ${ }^{4,5}$ Early treatment with statins can effectively improve lipid metabolism and inflammation indexes and reduce mortality. However, there are relatively few studies on gout complicated with carotid plaque. The purpose of this study was to provide more evidence to guide clinical treatment.

\section{METHODS}

Seventy patients with gout were admitted to our hospital from January 2014 to June 2017, including 46 males and 24 females. PASS software was used to calculate the sample size. A randomized, double-blind approach was used to divide the participants into the treatment group (35 cases, male (24 cases) and female (11 cases)) and the control group ( 35 cases, male ( 22 cases) and female (13 cases). There were no significant differences in general data between the two groups $(\mathrm{P}>0.05)$, as shown in Table-I.

The study was approved by the Institutional Ethics Committee of Affiliated Hospital of Hebei University (No. HDFY-LL-2020-090; date:23-072020), and written informed consent was obtained from all participants.

All patients met the 1981 American College of Rheumatology (ACR) gout classification criteria. ${ }^{3}$ (1) The articular fluid was confirmed to contain urate crystals possessing characteristic features, or (2) Confirmation of other markers by chemical methods or polarization microscopy, or (3) Six out of the following 12 findings: acute arthritis attacks occurring more than once; the inflammatory reaction peaked within one day; the onset of monoarthritis; visible joint redness; pain or swelling of the first metatarsophalangeal joint; an attack of the unilateral first metatarsophalangeal; the onset of unilateral tarsal arthritis; presence of a tophus; hyperuricemia; asymmetrical intraarticular swelling (confirmed by X-ray); subcortical cyst without erosion (confirmed by X-ray); and negative microbial culture from synovial fluid at the onset of arthritis.

Inclusion criteria: Patients between ages 18-65 years were initially treated for gout in the intermittent stage but had not been treated with hyaluronic acid medication, as well as if their uric acid levels were $\geq 480 \mu \mathrm{mol} / 1$ and their course of disease was $>2$ weeks. The patients were informed about the study and signed a consent form.

Exclusion criteria: (1) Patients with acute gout onset. (2) Patients receiving theophylline and other drugs to reduce uric acid salt. (3) Patients with cardiovascular and cerebrovascular diseases, endocrine diseases and neuropsychiatric diseases. (4) Patients who had an allergic condition allergy to febuxostat or atorvastatin or other contraindications. (5) Pregnant and lactating women.

Treatment group: Patients received febuxostat 40 $\mathrm{mg}$ once per day and atorvastatin $40 \mathrm{mg}$ once per day. Control group: Patients received febuxostat 40 $\mathrm{mg} /$ day and atorvastatin $20 \mathrm{mg} /$ day. The course of treatment was 90 days.

Observation indicators: The patients' TNF- $\mathrm{a}$, IL-1 $\beta$, and CRP levels were examined, as well as their ESRs and carotid plaques. TNF- $\alpha$ and IL$1 \beta$ were detected by ELISA, and the erythrocyte sedimentation rate (ESR) was detected by Westergren's method. C-reactive protein (CRP) was detected using a Beckman AU5800 analyzer and the supplier's original reagent. Carotid plaques were detected by GE S8 ultrasound. SPSS 19.0 statistical software was used. Statistical significance was determined for the differences between the two groups by $t$ test $(p<0.05)$.

\section{RESULTS}

After 90 days of treatment, serum TNF-a, IL$1 \beta$, and CRP levels, as well as HUA and total cholesterol (TC), decreased in both groups after treatment. There were significant differences observed ( $p<0.05)$. Table-II. Changes in carotid plaque: The carotid artery plaques in the two groups were significantly smaller after treatment $(\mathrm{P}<0.05)$. Table-III. Adverse reactions: There was no significant difference in adverse reactions between the two groups $(\mathrm{P}>0.05)$. Table-IV.

\section{DISCUSSION}

Gout is a type of acute and chronic inflammation and tissue damage caused by the deposition of

Table-I: Comparison of General Data of the Two Groups $(\bar{x} \pm s)$.

\begin{tabular}{lccc}
\hline Group & $n$ & Male/Female & Age \\
\hline Treatment group & 35 & $24 / 11$ & $47.2 \pm 6.5$ \\
Control group & 35 & $22 / 13$ & $48.6 \pm 7.1$ \\
\hline
\end{tabular}


Table-II: Changes in TNF- $\alpha$, IL-1 $\beta$, CRP, HUA and TC in the two groups before and after treatment $(\bar{x} \pm \mathrm{s}, \mathrm{n}=30)$.

\begin{tabular}{llccccc}
\hline Group & Time & TNF-a $(\mathrm{ng} / \mathrm{ml})$ & $I L-1 \beta(\mathrm{ng} / \mathrm{ml})$ & $\mathrm{CR}(\mathrm{mg} / \mathrm{L})$ & $\mathrm{HUA}(\mu \mathrm{mol} / \mathrm{L})$ & $\mathrm{TC}(\mathrm{mmol} / \mathrm{L})$ \\
\hline Control group & Before treatment & $40.83 \pm 9.24$ & $3.71 \pm 1.62$ & $15.8 \pm 6.2$ & $579 \pm 72$ & $6.36 \pm 1.28$ \\
& After treatment & $25.63 \pm 5.33 \Delta$ & $3.12 \pm 0.96 \Delta$ & $11.2 \pm 3.5 \Delta$ & $318 \pm 49$ & $3.69 \pm 0.94 \Delta$ \\
\multirow{2}{*}{ Treatment group } & Before treatment & $42.41 \pm 10.82$ & $3.58 \pm 1.86$ & $14.9 \pm 5.5$ & $588 \pm 67$ & $6.55 \pm 1.19$ \\
& After treatment & $22.79 \pm 4.51^{*} \Delta$ & $2.87 \pm 1.05^{*} \Delta$ & $9.6 \pm 2.8^{*} \Delta$ & $309 \pm 41$ & $2.92 \pm 0.87 \Delta$ \\
\hline
\end{tabular}

Compared with the same group before treatment,

$\Delta p<0.05$; compared with the control group ${ }^{*} p<0.05,{ }^{*}$ $p>0.05$.

Table-III: Color Doppler ultrasound of carotid artery plaques before and after treatment in the two groups $(\bar{x} \pm \mathrm{s}, \mathrm{n}=30)$.

\begin{tabular}{llccc}
\hline Group & Time & Patch size $\left(\mathrm{cm}^{2}\right)$ & Patch thickness $(\mathrm{mm})$ & Number of plaques (number) \\
\hline Control group & Before treatment & $0.081 \pm 0.026$ & $2.72 \pm 0.73$ & $3.72 \pm 0.89$ \\
& After treatment & $0.052 \pm 0.021 \Delta$ & $1.91 \pm 0.87 \Delta$ & $2.71 \pm 0.71 \Delta$ \\
Treatment group & Before treatment & $0.079 \pm 0.030$ & $2.81 \pm 0.86$ & $3.61 \pm 0.80$ \\
& After treatment & $0.041 \pm 0.017^{*} \Delta$ & $1.47 \pm 0.75^{*} \Delta$ & $2.36 \pm 0.68^{*} \Delta$ \\
\hline
\end{tabular}

monosodium urate in bone joints, kidneys and subcutaneous areas, and it is directly related to hyperuricemia caused by purine metabolic disorder and/or reduced uric acid excretion. ${ }^{6}$ During an acute attack, the main manifestations were inflammation, joint swelling, heat and pain and were usually relieved spontaneously within two weeks. After that, the patient has entered the intermission period between attacks, which is characterized by asymptomatic hyperuricemia. Previous reports have shown that there remains a slight chronic inflammatory state in the patient during the intermission period. It is presumed that uric acid plays an inflammatory role through the crystallization of monosodium urate (MSU) or soluble factors. Some studies have shown that MSU can stimulate the expression of TLR4, NLRP3, TNF- $\alpha$, and IL-1 $\beta .^{7}$ On the other hand, dissolved uric acid can enter cells and activate MAP kinase (P38 and ERK), which further activates NF- $\mathrm{KB}$, and induce the expression of inflammatory mediators such as MCP-1 and CRP. ${ }^{8}$ Among the implicated factors, TNF- $\alpha$, IL$1 \beta$ and CRP are important components of the chronic inflammatory environment.

Tumor necrosis factor-a (TNF- $\alpha$ ) is an earlydiscovered proinflammatory cytokine, and its biological effects have been studied extensively.
It is mainly produced by activated mononuclear macrophages and partially produced by $\mathrm{T}$ cells. It can promote lymphocyte activation and fibroblast proliferation and has effects that regulate immunity and mediate the inflammatory response. In addition, it also has effects on cytokines, chemokines, prostaglandins and metalloproteases and plays an important pathological role in various autoimmune diseases. ${ }^{9}$ Its role in the course of gout is relatively clear. TNF- $\alpha$ is the main inflammatory mediator in the body. Atherosclerosis is also, by nature, an inflammatory vascular disease. Therefore, in the early stages of atherosclerosis, TNF-a may get involved in the development of atherosclerotic plaques by destroying the integrity of vascular endothelial structure and function, transcription of the SIS protooncogene, proliferation of vascular smooth muscle cells, and the balance of coagulation and anticoagulation systems, etc. ${ }^{10-}$ ${ }^{11}$ At the same time, TNF-a can also activate the cytokine system, thereby activating the systemic inflammatory response and accelerating the formation of atherosclerosis. ${ }^{12}$ A considerable number of studies have shown that TNF-a is involved in the process of inflammation and development of atherosclerosis and plays a key role in promoting both. ${ }^{13,14}$

Table-IV: Color Doppler ultrasound of carotid artery plaque

before and after treatment in the two groups $(\bar{x} \pm \mathrm{s}, \mathrm{n}=30)$.

\begin{tabular}{lcccc}
\hline Group & Nausea & Headache & Vertigo & Total adverse reactions \\
\hline Control group & $2(5.7)$ & $1(2.9)$ & $1(2.9)$ & $4(11.4)$ \\
Treatment group & $1(2.9)$ & $2(5.7)$ & $2(5.7)$ & $5(14.3)$ \\
\hline
\end{tabular}


In 2006, Martinon and his students proposed that IL-1 $\beta$ and the NALP3 inflammasome are key factors in the inflammatory response. ${ }^{15}$ After the NLRP3 inflammasome is activated in gout patients, inactive IL-1 $\beta$ precursors can be cleaved into mature IL-1 $\beta$, further amplifying the inflammatory response of the body. At the same time, IL-1 $\beta$ is an important inflammatory factor in the occurrence and development of various metabolic diseases. ${ }^{16}$ Inflammation usually plays a very important role in the pathogenesis of various chronic diseases or states. For example, studies on atherosclerosis have found that cholesterol crystals can produce a large amount of IL-1 $\beta^{17,18}$ by activating the NALP3 inflammasome. Geng et al confirmed that IL-1 $\beta$ is an atherogenic cytokine and can promote apoptosis of normal vascular smooth muscle cells; inflammation plays an important role in gout and atherosclerosis. ${ }^{19}$ The two are intrinsically linked.

Increasing data show that uric acid is involved in many aspects of the occurrence, progression and deterioration of atherosclerosis. Therefore, it is very important to reduce uric acid while improving atherosclerosis and reducing inflammatory reactions. Febuxostat is a new xanthine oxidase inhibitor that has an obvious inhibitory effect on the oxidation and reduction forms of the enzyme and can noticeably reduce the level of uric acid. At the same time, it can also improve the inflammatory state of the body. Atorvastatin is an HMG-CoA reductase inhibitor that has a significant lipid-lowering effect along with low toxicity and relative safety. In recent years, atorvastatin has also been found to be anti-inflammatory and anti-atherosclerotic. It can effectively reduce the levels of inflammatory factors such as high-sensitivity $\mathrm{C}$-reactive protein and TNF- $\alpha$ in diabetic patients in a short period of time. Several studies have shown that atorvastatin can affect the expression of nuclear factor $\mathrm{kB}$, TLR4 and its downstream signals and MMP9, inhibit inflammatory reactions and stabilize atherosclerotic plaques in various ways. ${ }^{20,22}$

The results showed that TNF- $\alpha$, IL-1 $\beta$, CRP and other inflammatory indexes in the treatment group were significantly lower than those in the control group. Atherosclerotic plaque area decreased, indicating that double doses of atorvastatin combined with febuxostat can better reduce inflammatory reactions in patients with gout and carotid atherosclerosis. The curative effect of a double dose of atorvastatin was fully affirmed, and the number of cases of nausea, headache, dizziness and insomnia in the treatment group was not different from that in the control group. These results showed that the double dose of atorvastatin in the test group did not increase the incidence of adverse reactions.

Limitations of the study: Dietary factors and other metabolic related diseases may have an impact on the results of the study. Therefore, a multi factor analysis will be carried out in the next step to observe the correlation between various factors and the research indicators.

\section{CONCLUSION}

In summary, the double dose of atorvastatin combined with febuxostat in the treatment of patients with gout and carotid atherosclerosis can effectively reduce uric acid, improve the inflammatory state in patients, reduce carotid plaque and have good clinical benefits.

\section{Declaration of conflicts of interest: None.}

Funding: Scientific research project of Traditional Chinese Medicine in Hebei Administration of Traditional Chinese Medicine (Project No: 2018 148).

\section{REFERENCES}

1. Conen D, Wietlisbach V, Bovet P, Shamlaye C, Riesen W, Paccaud F, et al. Prevalence of hyperuricemia and relation of serum uric acid with cardiovascular risk factors in a developing country. BMC Public Health. 2004;4:9. doi:10.1186/1471-2458-4-9

2. Dincer HE, Dincer AP, Levinson DJ. Asymptomatic hyperuricemia: to treat or not to treat. Cleve Clin J Med. 2002;69(8). doi:10.3949/ccjm.69.8.594.

3. Puig JG, de Miguel E, Castillo MC, Rocha AL, Martínez MA, Torres RJ. Asymptomatic hyperuricemia: impact of ultrasonography. Nucleosides Nucleotides Nucleic Acids. 2008;27(6):592-595. doi:10.1080/15257770802136040

4. Sodergren A, Karp K, Boman K, Eriksson C, Lundstrom E, Smedby T, et al. Atherosclerosis in early rheumatoid arthritis: very early endothelial activation and rapid progression of intima media thickness. Arthritis Res Ther. 2010;12(4):R158. doi: 10.1186/ar3116

5. Kang EH, Liao KP, Kim SC. Cardiovascular Safety of Biologics and JAK Inhibitors in Patients with Rheumatoid Arthritis. Curr Rheumatol Rep. 2018;20(7):42. doi:10.1007/ s11926-018-0752-2

6. Shiramoto M, Liu S, Shen Z, Yan X, Yamamoto A, Gillen $M$, et al. Verinurad combined with febuxostat in Japanese adults with gout or asymptomatic hyperuricaemia: a phase 2a, open-label study. Rheumatology (Oxford). 2018;57(9):1602-1610. doi:10.1093/rheumatology/key100 
7. Piancone F, Saresella M, Marventano I, La Rosa F, Santangelo MA, Caputo D, et al. Monosodium Urate Crystals Activate the Inflammasome in Primary Progressive Multiple Sclerosis. Front Immunol. 2018;9:983. doi:10.3389/fimmu.2018.00983

8. Sautin YY, Nakagawa T, Zharikov S, Johnson RJ. Adverse effects of the classic antioxidant uric acid in adipocytes: NADPH oxidase-mediated oxidative/nitrosative stress [published correction appears in Am J Physiol Cell Physiol. 2010 Sep;299(3):C726]. Am J Physiol Cell Physiol. 2007;293(2):C584-C596. doi:10.1152/ajpcell.00600.2006

9. Malaise MG, Chapelier N, Dewael T, Leonori L, Leroy M, Mailleux E, et al. Remarkable medical advances in rheumatology: May be... Rev Med Liege. 2020;75(56):369-375.

10. Valgimigli M, Merli E, Malagutti $P$, Soukhomovskaia $O$, Cicchitelli G, Antelli A, et al. Hydroxyl radical generation, levels of tumor necrosis factor-alpha, and progression to heart failure after acute myocardial infarction. J Am Coll Cardiol. 2004;43(11):2000-2008. doi:10.1016/j. jacc.2004.01.036

11. Jovinge $\mathrm{S}$, Hultgårdh-Nilsson A, Regnström J, Nilsson J. Tumor necrosis factor-alpha activates smooth muscle cell migration in culture and is expressed in the balloon-injured rat aorta. Arterioscler Thromb Vasc Biol. 1997;17(3):490497. doi:10.1161/01.atv.17.3.490

12. Curran MP. Amlodipine/Atorvastatin: a review of its use in the treatment of hypertension and dyslipidaemia and the prevention of cardiovascular disease. Drugs. 2010;70(2):191-213. doi:10.2165/11204420-000000000-00000

13. Chen YY, Hsu MJ, Hsieh CY, Lee LW, Chen ZC, Sheu JR. Andrographolide inhibits nuclear factor- $\mathrm{kB}$ activation through JNK-Akt-p65 signaling cascade in tumor necrosis factor-a-stimulated vascular smooth muscle cells. Scientific World J. 2014;2014:130381. doi:10.1155/2014/130381

14. van Kuilenburg J, Lappegard KT, Sexton J, Plesiewicz I, Lap P, Bouwels L, et al. Persisting thrombin activity in elderly patients with atrial fibrillation on oral anticoagulation is decreased by anti-inflammatory therapy with intensive cholesterol-lowering treatment. J Clin Lipidol. 2011;5(4):273-280. doi:10.1016/j.jacl.2011.05.003

15. Martinon F, Pétrilli V, Mayor A, Tardivel A, Tschopp J. Gout-associated uric acid crystals activate the NALP3 inflammasome. Nature. 2006;440(7081):237-241. doi:10.1038/nature04516.
16. Shaw PJ, McDermott MF, Kanneganti TD. Inflammasomes and autoimmunity. Trends Mol Med. 2011;17(2):57-64. doi:10.1016/j.molmed.2010.11.001

17. Rajamaki K, Lappalainen J, Oorni K, Valimaki E, Matikainen $\mathrm{S}$, Kovanen PT, et al. Cholesterol crystals activate the NLRP3 inflammasome in human macrophages: a novel link between cholesterol metabolism and inflammation. PLoS One. 2010;5(7):e11765. doi:10.1371/journal.pone.0011765

18. Duewell $\mathrm{P}$, Kono $\mathrm{H}$, Rayner $\mathrm{KJ}$, Sirois $\mathrm{CM}$, Vladimer G, Bauernfeind FG, et al. NLRP3 inflammasomes are required for atherogenesis and activated by cholesterol crystals [published correction appears in Nature. 2010;466(7306):652]. Nature. 2010;464(7293):1357-1361. doi:10.1038/nature08938

19. Geng YJ, Wu Q, Muszynski M, Hansson GK, Libby P. Apoptosis of vascular smooth muscle cells induced by in vitro stimulation with interferon-gamma, tumor necrosis factor-alpha, and interleukin-1 beta. Arterioscler Thromb Vasc Biol. 1996;16(1):19-27. doi:10.1161/01.atv.16.1.19

20. Geng J, Xu H, Fu W, Yu X, Xu G, Cao H, et al. Rosuvastatin protects against endothelial cell apoptosis in vitro and alleviates atherosclerosis in ApoE-/- mice by suppressing endoplasmic reticulum stress. Exp Ther Med. 2020;20(1):550-560. doi:10.3892/etm.2020.8733

21. Jin Y, Qiu C, Zheng Q, Liu L, Liu Z, Wang Y. Efficacy of different doses of atorvastatin treatment on serum levels of 8-hydroxy-guanin (8-OHdG) and cardiac function in patients with ischemic cardiomyopathy. Pak J Med Sci. 2015;31(1):37-42. doi:10.12669/pjms.311.5840

22. Buraczynska K, Kurzepa J, Ksiazek A, Buraczynska M, Rejdak K. Matrix Metalloproteinase-9 (MMP-9) Gene Polymorphism in Stroke Patients. Neuromolecular Med. 2015;17(4):385-390. doi:10.1007/s12017-015-8367-5

\section{Authors' Contributions:}

ZZ \& MHX: Designed this study and prepared this manuscript, and are responsible and accountable for the accuracy or integrity of the work. LS: Collected and analyzed clinical data. FW: Significantly revised this manuscript. ZZ \& MX: contribute to this work equally. 